Article

\title{
Multi-Term Fractional Degenerate Evolution Equations and Optimal Control Problems
}

\author{
Marina Plekhanova ${ }^{1,2, *(D)}$ and Guzel Baybulatova 2 (D) \\ 1 Department of Computational Mechanics, South Ural State University (National Research University), \\ Lenin Av. 76, Chelyabinsk 454001, Russia \\ 2 Department of Mathematical Analysis, Chelyabinsk State University, Kashirin Brothers St. 129, \\ Chelyabinsk 454001, Russia; baybulatova_g_d@mail.ru \\ * Correspondence: mariner79@mail.ru; Tel.: +7-952-514-1719
}

Received: 5 February 2020; Accepted: 17 March 2020; Published: 1 April 2020

check for updates

Abstract: A theorem on unique solvability in the sense of the strong solutions is proved for a class of degenerate multi-term fractional equations in Banach spaces. It applies to the deriving of the conditions on unique solution existence for an optimal control problem to the corresponding equation. Obtained results are used to an optimal control problem study for a model system which is described by an initial-boundary value problem for a partial differential equation.

Keywords: fractional derivative; multi-term fractional equation; degenerate evolution equation; optimal control

\section{Introduction}

We consider an optimal control problem for the multi-term fractional equation

$$
L D_{t}^{\alpha} x(t)=M x(t)+\sum_{k=1}^{n} N_{k}(t) D_{t}^{\alpha_{k}} x(t)+B u(t), \quad t \in\left(t_{0}, T\right) .
$$

Here $\mathcal{X}, \mathcal{Y}, \mathcal{U}$ are Banach spaces, $L: \mathcal{X} \rightarrow \mathcal{Y}$ is a continuous operator, a linear closed operator $M$ with a dense domain in $\mathcal{X}$ acts into $\mathcal{Y}$, linear and continuous operator $B$ acts on control function $u$ from $\mathcal{U}$ into $\mathcal{Y}$. Operators $N_{k}(t)$ are linear and continuous for every $t \in\left(t_{0}, T\right), k=1,2, \ldots, n$. We mean the Gerasimov-Caputo derivatives under notations $D_{t}^{\alpha}$ and $D_{t}^{\alpha_{k}}$ with $0 \leq \alpha_{1}<\alpha_{2}<\cdots<\alpha_{n} \leq m-1<$ $\alpha \leq m \in \mathbb{N}$. Equations, which are not solved with respect to the highest order derivative with respect to time, are often called Sobolev type equations [1,2]. Moreover, if (1) contains the operator $L$ with a nontrivial kernel $\operatorname{ker} L \neq\{0\}$, it often called degenerate evolution equation or degenerate equation [3]. Here we shall consider this case.

Equations of form (1) frequently encountered in applications (see references below). The natural initial conditions for degenerate evolution Equation (1) are

$$
(P x)^{(k)}\left(t_{0}\right)=x_{k}, k=0,1, \ldots, m-1,
$$

where $P$ is the projector along the degeneration space of the equation. We require that control functions have to belong the admissible controls set

$$
u \in \mathcal{U}_{\partial}
$$

where $\mathcal{U}_{\partial}$ is a nonempty closed convex set of a control functions space $L_{q}\left(t_{0}, T ; \mathcal{U}\right)$. The cost functional has the form

$$
J(x, u)=\left\|x-x_{d}\right\|_{C^{m-1}\left(\left[t_{0}, T\right] ; \mathcal{X}\right)}+\left\|D_{t}^{\alpha} x-D_{t}^{\alpha} x_{d}\right\|_{L_{q}\left(t_{0}, T ; \mathcal{X}\right)}^{q}+\delta\left\|u-u_{d}\right\|_{L_{q}\left(t_{0}, T ; \mathcal{U}\right)}^{q} \rightarrow \text { inf, }
$$


where $q>1, \delta>0, x_{d}$ and $u_{d}$ are given functions. We are going to establish solvability conditions of problem (1)-(4).

In recent decades, fractional integro-differential calculus has become one of the most important tools for solving mathematical modeling problems [4-8]. On the other hand various issues of the control theory, including unique solvability, are of interest to many authors. However, not many papers deal with control problems for fractional differential equations, see [9-12] and references therein. The present paper is a continuation of the authors' works on optimal control problems for the equations with a degenerate operator at the highest-order time-fractional derivative [13-19].

In the second section we give the definition of the Gerasimov-Caputo fractional derivative and a result about the existence of a unique strong solution of the Cauchy problem for a semilinear equation which is solved with respect to the highest-order fractional derivative. The third section contains the proof of the unique solvability in the sense of the strong solutions for a class of initial problems of form (1) and (2). Here we used the theory of the degenerate evolution equations (see works $[2,20,21])$. In the fourth section the result on the existence of a unique strong solution for problem (1), (2) is applied to the proof of the optimal control existence for (1)-(4). The last section of the paper illustrates the obtained abstract results on an example of an initial-boundary value problem for a partial differential equation.

\section{Solvability of Nondegenerate Semilinear Equation}

Let $\mathcal{Z}$ be a Banach space. Denote $g_{\delta}(t):=\Gamma(\delta)^{-1} t^{\delta-1}, \tilde{g}_{\delta}(t):=\Gamma(\delta)^{-1}\left(t-t_{0}\right)^{\delta-1}, J_{t}^{\delta} h(t):=$ $\int_{t_{0}}^{t} g_{\delta}(t-s) h(s) d s$ for $\delta>0, t>0$. Let $m-1<\alpha \leq m \in \mathbb{N}, D_{t}^{m}$ be the usual derivative of the order $m \in \mathbb{N}, J_{t}^{0}$ be the identical operator. The Gerasimov-Caputo derivative of a function $h$ is (see [22] (p. 11))

$$
D_{t}^{\alpha} h(t)=D_{t}^{m} J_{t}^{m-\alpha}\left(h(t)-\sum_{k=0}^{m-1} h^{(k)}\left(t_{0}\right) \tilde{g}_{k+1}(t)\right), \quad t \geq t_{0} .
$$

Consider the Cauchy problem

$$
z^{(k)}\left(t_{0}\right)=z_{k}, \quad k=0,1, \ldots, m-1,
$$

for the nonlinear differential equation

$$
D_{t}^{\alpha} z(t)=A z(t)+B\left(t, D_{t}^{\alpha_{1}} z(t), D_{t}^{\alpha_{2}} z(t), \ldots, D_{t}^{\alpha_{n}} z(t)\right)
$$

where $A \in \mathcal{L}(\mathcal{Z})$, i.e., linear and continuous operator from $\mathcal{Z}$ to $\mathcal{Z}, 0 \leq \alpha_{1}<\alpha_{2}<\cdots<\alpha_{n} \leq m-1<$ $\alpha \leq m \in \mathbb{N}, n \in \mathbb{N}$, a nonlinear operator $B:\left(t_{0}, T\right) \times \mathcal{Z}^{n} \rightarrow \mathcal{Z}$ is a Caratheodory mapping, i.e., for arbitrary $z_{1}, z_{2}, \ldots, z_{n} \in \mathcal{Z}$ it defines a measurable mapping on $\left(t_{0}, T\right)$ and for almost all $t \in\left(t_{0}, T\right)$ it is continuous in $z_{1}, z_{2}, \ldots, z_{n} \in \mathcal{Z}$.

A strong solution of problem (5), (6) is a function $z \in C^{m-1}\left(\left[t_{0}, T\right] ; \mathcal{Z}\right)$, such that $J_{t}^{m-\alpha}\left(z-\sum_{k=0}^{m-1} z^{(k)}\left(t_{0}\right) \tilde{g}_{k+1}\right) \in W_{q}^{m}\left(t_{0}, T ; \mathcal{Z}\right)$, equalities (5) and (6) for almost all $t \in\left(t_{0}, T\right)$ are true. Here we use some $q>1$.

Denote as $\bar{x}=\left(x_{1}, x_{2}, \ldots, x_{n}\right)$ a set of $n$ elements. We shall say that operator $B:\left(t_{0}, T\right) \times \mathcal{Z}^{n} \rightarrow \mathcal{Z}$ is uniformly Lipschitz continuous in $\bar{x} \in \mathcal{Z}^{n}$, if there exists $l>0$, such that the inequality $\| B(t, \bar{x})-$ $B(t, \bar{y})\left\|_{\mathcal{Z}} \leq l \sum_{k=1}^{n}\right\| x_{k}-y_{k} \|_{\mathcal{Z}}$ is true for almost all $t \in\left(t_{0}, T\right)$ and for all $\bar{x}, \bar{y} \in \mathcal{Z}^{n}$.

Lemma 1 ([20]). Let $l-1<\beta \leq l \in \mathbb{N}, t>t_{0}$. Then

$$
\exists C_{\beta}>0 \quad \forall h \in C^{l}\left(\left[t_{0}, t\right] ; \mathcal{Z}\right) \quad\left\|D_{t}^{\beta} h\right\|_{C\left(\left[t_{0}, t\right] ; \mathcal{Z}\right)} \leq C_{\beta}\|h\|_{C^{l}\left(\left[t_{0}, t\right] ; \mathcal{Z}\right)} .
$$


Theorem 1 ([23]). Suppose that $q>(\alpha-m+1)^{-1}, z_{0}, z_{1}, \ldots, z_{m-1} \in \mathcal{Z}, A \in \mathcal{L}(\mathcal{Z}), B:\left(t_{0}, T\right) \times \mathcal{Z}^{n} \rightarrow$ $\mathcal{Z}$ is Caratheodory mapping, which is uniformly Lipschitz continuous in $\bar{x}$, at all $y_{1}, y_{2}, \ldots, y_{n} \in \mathcal{Z}$ and almost everywhere on $\left(t_{0}, T\right)$ inequality

$$
\left\|B\left(t, y_{1}, y_{2}, \ldots, y_{n}\right)\right\|_{\mathcal{Z}} \leq a(t)+c \sum_{k=1}^{n}\left\|y_{k}\right\|_{\mathcal{Z}}
$$

is valid for some $a \in L_{q}\left(t_{0}, T ; \mathbb{R}\right), c>0$. Then problem (5), (6) has a unique strong solution on $\left(t_{0}, T\right)$.

\section{Degenerate Multi-Term Linear Equation}

Let $\mathcal{X}$ and $\mathcal{Y}$ be Banach spaces. As $\mathcal{L}(\mathcal{X} ; \mathcal{Y})$ we denote the space of all linear continuous operators, which act from the space $\mathcal{X}$ to $\mathcal{Y}$. Denote by $\mathcal{C l}(\mathcal{X} ; \mathcal{Y})$ the set of all linear closed operators with a dense domain in $\mathcal{X}$ and with an image in $\mathcal{Y}$. Suppose that $L \in \mathcal{L}(\mathcal{X} ; \mathcal{Y}), M \in \mathcal{C l}(\mathcal{X} ; \mathcal{Y})$, denote by $D_{M}$ the domain of $M$, endowed by the graph norm $\|\cdot\|_{D_{M}}:=\|\cdot\|_{\mathcal{X}}+\|M \cdot\|_{\mathcal{Y}}$.

Define $L$-resolvent set $\rho^{L}(M):=\left\{\mu \in \mathbf{C}:(\mu L-M)^{-1} \in \mathcal{L}(\mathcal{Y} ; \mathcal{X})\right\}$ of an operator $M$ and its $L$-spectrum $\sigma^{L}(M):=\mathbf{C} \backslash \rho^{L}(M)$, and denote $R_{\mu}^{L}(M):=(\mu L-M)^{-1} L, L_{\mu}^{L}:=L(\mu L-M)^{-1}$.

An operator $M$ is called $(L, \sigma)$-bounded, if

$$
\exists a>0 \quad \forall \mu \in \mathbb{C} \quad(|\mu|>a) \Rightarrow\left(\mu \in \rho^{L}(M)\right) .
$$

Under the condition of $(L, \sigma)$-boundedness of operator $M$ we have the projections

$$
P:=\frac{1}{2 \pi i} \int_{\gamma} R_{\mu}^{L}(M) d \mu \in \mathcal{L}(\mathcal{X}), Q:=\frac{1}{2 \pi i} \int_{\gamma} L_{\mu}^{L}(M) d \mu \in \mathcal{L}(\mathcal{Y}),
$$

where $\gamma=\{\mu \in \mathbb{C}:|\mu|=r>a\}$ (see [2] (pp. 89-90)). Put $\mathcal{X}^{0}:=\operatorname{ker} P, \mathcal{X}^{1}:=\operatorname{im} P, \mathcal{Y}^{0}:=\operatorname{ker} Q$, $\mathcal{Y}^{1}:=\operatorname{im} Q$. Denote by $L_{k}\left(M_{k}\right)$ the restriction of the operator $L(M)$ on $\mathcal{X}^{k}\left(D_{M_{k}}:=D_{M} \cap \mathcal{X}^{k}\right), k=0,1$.

Theorem 2 ([2] (pp. 90-91)). Let an operator $M$ be $(L, \sigma)$-bounded. Then

(i) $M_{1} \in \mathcal{L}\left(\mathcal{X}^{1} ; \mathcal{Y}^{1}\right), M_{0} \in \mathcal{C l}\left(\mathcal{X}^{0} ; \mathcal{Y}^{0}\right), L_{k} \in \mathcal{L}\left(\mathcal{X}^{k} ; \mathcal{Y}^{k}\right), k=0,1$;

(ii) there exist operators $M_{0}^{-1} \in \mathcal{L}\left(\mathcal{Y}^{0} ; \mathcal{X}^{0}\right), L_{1}^{-1} \in \mathcal{L}\left(\mathcal{Y}^{1} ; \mathcal{X}^{1}\right)$.

Denote $\mathbb{N}_{0}:=\{0\} \cup \mathbb{N}, G:=M_{0}^{-1} L_{0}$. For $p \in \mathbb{N}_{0}$ operator $M$ is called $(L, p)$-bounded, if it is $(L, \sigma)$-bounded, $G^{p} \neq 0, G^{p+1}=0$.

Let $n \in \mathbb{N}, N_{k}:\left(t_{0}, T\right) \rightarrow \mathcal{L}(\mathcal{X} ; \mathcal{Y}), k=1,2, \ldots, n, 0 \leq \alpha_{1}<\alpha_{2}<\cdots<\alpha_{n} \leq m-1 \in \mathbb{N}_{0}$. Consider the degenerate multi-term linear equation

$$
L D_{t}^{\alpha} x(t)=M x(t)+\sum_{k=1}^{n} N_{k}(t) D_{t}^{\alpha_{k}} x(t)+f(t), \quad t \in\left(t_{0}, T\right) .
$$

Fix a constant $q>1$. Strong solution on $\left(t_{0}, T\right)$ of this equation is a function $x \in C^{m-1}\left(\left[t_{0}, T\right] ; \mathcal{X}\right) \cap$ $L_{q}\left(t_{0}, T ; D_{M}\right)$, such that

$$
J_{t}^{m-\alpha}\left(x-\sum_{k=0}^{m-1} x^{(k)}\left(t_{0}\right) \tilde{g}_{k+1}\right) \in W_{q}^{m}\left(t_{0}, T ; \mathcal{Y}\right)
$$

and almost everywhere on $\left(t_{0}, T\right)$ equality (8) holds.

A solution of the generalized Showalter-Sidorov problem

$$
(P x)^{(k)}\left(t_{0}\right)=x_{k}, \quad k=0,1, \ldots, m-1,
$$


to Equation (8) is a solution of the equation, such that conditions (9) are true. Note here that $P x=$ $L_{1}^{-1} L_{1} P x=L_{1}^{-1} Q L x$, hence the smoothness of $P x$ is not less the smoothness of $L x$, since $L_{1}^{-1} Q \in$ $\mathcal{L}(\mathcal{Y} ; \mathcal{X})$ due to Theorem 2 .

Lemma 2 ([19]). Let $H \in \mathcal{L}(\mathcal{X})$ be a nilpotent operator of a power $p \in \mathbb{N}_{0}$, a function $h:\left[t_{0}, T\right] \rightarrow$ $\mathcal{X},\left(H D_{t}^{\alpha}\right)^{l} h \in C^{m-1}\left(\left[t_{0}, T\right] ; \mathcal{X}\right)$ and $D_{t}^{\alpha}\left(H D_{t}^{\alpha}\right)^{l} h \in L_{q}\left(t_{0}, T ; \mathcal{X}\right)$ for $l=0,1, \ldots, p$. Then the equation $H D^{\alpha} x(t)=x(t)+h(t)$ has a unique strong solution. Moreover, it has the form $x(t)=-\sum_{l=0}^{p}\left(H D^{\alpha}\right)^{l} h(t)$.

Theorem 3. Let $q>(\alpha-m+1)^{-1}, p \in \mathbb{N}_{0}$, an operator $M$ be $(L, p)$-bounded, mappings $N_{k}:\left(t_{0}, T\right) \rightarrow$ $\mathcal{L}(\mathcal{X} ; \mathcal{Y})$ be measurable, essentially bounded on $\left(t_{0}, T\right), \operatorname{im}_{k}(t) \subset \mathcal{Y}^{1}$ for almost all $t \in\left(t_{0}, T\right), k=$ $1,2, \ldots, n, Q f \in L_{q}\left(t_{0}, T ; \mathcal{Y}\right)$, for all $l=0,1, \ldots, p$ there exist $\left(G D_{t}^{\alpha}\right)^{l} M_{0}^{-1}(I-Q) f \in C^{m-1}\left(\left[t_{0}, T\right] ; \mathcal{X}\right)$, $D_{t}^{\alpha}\left(G D_{t}^{\alpha}\right)^{l} M_{0}^{-1}(I-Q) f \in L_{q}\left(t_{0}, T ; \mathcal{X}\right) ; x_{0}, x_{1}, \ldots, x_{m-1} \in \mathcal{X}^{1}$. Then problem (8), (9) has a unique strong solution.

Proof. The mapping

$$
x(\cdot) \rightarrow \sum_{k=1}^{n} N_{k}(\cdot) D_{t}^{\alpha_{k}} x(\cdot)
$$

acts from $C^{m-1}\left(\left[t_{0}, T\right] ; \mathcal{X}\right)$ into the space $L_{q}\left(t_{0}, T ; \mathcal{Y}\right)$ according to the theorem conditions. By the fact $\operatorname{im}_{k} \subset \mathcal{Y}^{1}$ we have $(I-Q) N_{k} \equiv 0, Q N_{k} \equiv N_{k}, k=1,2, \ldots, n$. Equation (8) after the action of the operator $M_{0}^{-1}(I-Q)$ has the form

$$
D_{t}^{\alpha} G w(t)=w(t)+M_{0}^{-1}(I-Q) f(t)
$$

where $w(t)=(I-P) x(t)$. Since the operator $G$ is nilpotent and due to Lemma 2, the unique solution of this equation has the form

$$
w(t)=-\sum_{l=0}^{p}\left(G D_{t}^{\alpha}\right)^{l} M_{0}^{-1}(I-Q) f(t)
$$

Note that $w \in C^{m-1}\left(\left[t_{0}, T\right] ; \mathcal{X}\right), D_{t}^{\alpha} w \in L_{q}\left(t_{0}, T ; \mathcal{X}\right)$, and

$$
M w=-(I-Q) f-L D_{t}^{\alpha} \sum_{l=0}^{p-1}\left(G D_{t}^{\alpha}\right)^{l} M_{0}^{-1}(I-Q) f \in L_{q}\left(t_{0}, T ; \mathcal{Y}\right)
$$

The next step is to prove the unique strong solution existence of the Cauchy problem

$$
\begin{gathered}
D_{t}^{\alpha} v(t)=S_{1} v(t)+L_{1}^{-1} \sum_{k=1}^{n} N_{k}(t) D_{t}^{\alpha_{k}}(v(t)+w(t))+L_{1}^{-1} Q f(t), \\
v^{(k)}\left(t_{0}\right)=x_{k}, k=0,1, \ldots, m-1,
\end{gathered}
$$

where $v(t)=P x(t), S_{1}=L_{1}^{-1} M_{1} \in \mathcal{L}\left(\mathcal{X}^{1}\right)$ due to Theorem 2. This problem is obtained from (8), (9) after the action of the continuous operator $L_{1}^{-1} Q$. Here the operator

$$
B\left(t, v_{0}, v_{1}, \ldots, v_{n}\right)=L_{1}^{-1} \sum_{k=1}^{n} N_{k}(t)\left(v_{k}+D_{t}^{\alpha_{k}} w(t)\right)+L_{1}^{-1} Q f(t)
$$

satisfies the conditions of Theorem 1. Indeed, let

$$
b:=\max _{k=1, \ldots, n} \operatorname{esssup} \underset{t \in\left(t_{0}, T\right)}{ }\left\|N_{k}(t)\right\|_{\mathcal{L}(\mathcal{X} ; \mathcal{Y})}
$$


then the operator $B$ is uniformly Lipschitz continuous in $\bar{v}$ with the constant $l=b\left\|L_{1}^{-1}\right\|_{\mathcal{L}(\mathcal{Y} ; \mathcal{X})}$ and it satisfies inequality (7) with $c=l$,

$$
a=L_{1}^{-1} \sum_{k=1}^{n} N_{k} D_{t}^{\alpha_{k}} w+L_{1}^{-1} Q f \in L_{q}\left(t_{0}, T ; \mathcal{X}\right)
$$

due to Lemma 1. Thus, by Theorem 1 we obtain the required. A unique solution of problem (8), (9) has the form $x(t)=v(t)+w(t)$.

\section{Optimal Control Problem}

Let $N_{k}:\left(t_{0}, T\right) \rightarrow \mathcal{L}(\mathcal{X} ; \mathcal{Y}), k=1,2, \ldots, n \in \mathbb{N}, 0 \leq \alpha_{1}<\alpha_{2}<\cdots<\alpha_{n} \leq m-1<\alpha \leq m \in \mathbb{N}$. Consider an optimal control problem for a degenerate multi-term linear equation

$$
\begin{gathered}
L D_{t}^{\alpha} x(t)=M x(t)+\sum_{k=1}^{n} N_{k}(t) D_{t}^{\alpha_{k}} x(t)+B u(t), \quad t \in\left(t_{0}, T\right), \\
(P x)^{(k)}\left(t_{0}\right)=x_{k}, k=0,1, \ldots, m-1, \\
u \in \mathcal{U}_{\partial}, \\
J(x, u)=\left\|x-x_{d}\right\|_{C^{m-1}\left(\left[t_{0}, T\right] ; \mathcal{X}\right)}+\left\|D_{t}^{\alpha} x-D_{t}^{\alpha} x_{d}\right\|_{L_{q}\left(t_{0}, T ; \mathcal{X}\right)}^{q}+\delta\left\|u-u_{d}\right\|_{L_{q}\left(t_{0}, T ; \mathcal{U}\right)}^{q} \rightarrow \text { inf, }
\end{gathered}
$$

where $\mathcal{U}_{\partial}$ is a subset of $L_{q}\left(t_{0}, T ; \mathcal{U}\right)$ of admissible controls, $J$ is the cost functional, $x_{d} \in C^{m-1}\left(\left[t_{0}, T\right] ; \mathcal{X}\right)$, such that $D_{t}^{\alpha} x_{d} \in L_{q}\left(t_{0}, T ; \mathcal{X}\right), u_{d} \in L_{q}\left(t_{0}, T ; \mathcal{U}\right)$ are given, $\delta>0$.

Introduce the spaces at $q>1$

$$
\begin{gathered}
\mathcal{Q}_{\alpha, q}\left(t_{0}, T ; \mathcal{X}\right):=\left\{z \in C^{m-1}\left(\left[t_{0}, T\right] ; \mathcal{X}\right): J_{t}^{m-\alpha}\left(z-\sum_{k=0}^{m-1} z^{(k)}\left(t_{0}\right) \tilde{g}_{k+1}\right) \in W_{q}^{m}\left(t_{0}, T ; \mathcal{X}\right)\right\}, \\
\mathcal{Z}_{\alpha, q}\left(t_{0}, T ; \mathcal{X}\right):=\left\{z \in C^{m-1}\left(\left[t_{0}, T\right] ; \mathcal{X}\right) \cap L_{q}\left(t_{0}, T ; D_{M}\right): J_{t}^{m-\alpha}\left(z-\sum_{k=0}^{m-1} z^{(k)}\left(t_{0}\right) \tilde{g}_{k+1}\right) \in W_{q}^{m}\left(t_{0}, T ; \mathcal{X}\right)\right\} .
\end{gathered}
$$

Lemma $3([13,14]) . \mathcal{Q}_{\alpha, q}\left(t_{0}, T ; \mathcal{X}\right)$ and $\mathcal{Z}_{\alpha, q}\left(t_{0}, T ; \mathcal{X}\right)$ are Banach spaces with the norms $\|x\|_{\mathcal{Q}_{\alpha, q}\left(t_{0}, T ; \mathcal{X}\right)}=$ $\|x\|_{C^{m-1}\left(\left[t_{0}, T\right] ; \mathcal{X}\right)}+\left\|D_{t}^{\alpha} x\right\|_{L_{q}\left(t_{0}, T ; \mathcal{X}\right)},\|x\|_{\mathcal{Z}_{\alpha, q}\left(t_{0}, T ; \mathcal{X}\right)}=\|x\|_{L_{q}\left(t_{0}, T ; D_{M}\right)}+\|x\|_{C^{m-1}\left(\left[t_{0}, T\right] ; \mathcal{X}\right)}+\left\|D_{t}^{\alpha} x\right\|_{L_{q}\left(t_{0}, T ; \mathcal{X}\right)}$ respectively.

Introduce the continuous operator $\gamma_{0}: C\left(\left[t_{0}, T\right] ; \mathcal{X}\right) \rightarrow \mathcal{X}, \gamma_{0} x=x\left(t_{0}\right)$.

Admissible pairs set $\mathcal{W}$ of problem (10)-(13) is a set of such pairs $(x, u)$, that $u \in \mathcal{U}_{\partial}, x \in$ $\mathcal{Z}_{\alpha, q}\left(t_{0}, T ; \mathcal{X}\right)$ is a strong solution of (10), (11). To solve problem (10)-(13) means to find the set of pairs $(\hat{x}, \hat{u}) \in \mathcal{W}$, which minimize the cost functional, i.e., $J(\hat{x}, \hat{u})=\inf _{(x, u) \in \mathcal{W}} J(x, u)$.

Theorem 4. Let $q>(\alpha-m+1)^{-1}, p \in \mathbb{N}_{0}$, an operator $M$ be $(L, p)$-bounded, mappings $N_{k}:\left(t_{0}, T\right) \rightarrow$ $\mathcal{L}(\mathcal{X} ; \mathcal{Y})$ be measurable, essentially bounded on $\left(t_{0}, T\right), \operatorname{im}_{k}(t) \subset \mathcal{Y}^{1}$ for almost all $t \in\left(t_{0}, T\right), k=$ $1,2, \ldots, n$. Assume that $\mathcal{U}_{\partial}$ is a non-empty closed convex subset in $L_{q}\left(t_{0}, T ; \mathcal{U}\right)$, there exists such $u_{0} \in \mathcal{U}_{\partial}$, that $\left(G D^{\alpha}\right)^{l} M_{0}^{-1}(I-Q) B u_{0} \in C^{m-1}\left(\left[t_{0}, T\right] ; \mathcal{X}\right), D^{\alpha}\left(G D^{\alpha}\right)^{l} M_{0}^{-1}(I-Q) B u_{0} \in L_{q}\left(t_{0}, T ; \mathcal{X}\right)$ at $l=0,1, \ldots, p$. Then problem (10)-(13) has a unique solution $(\hat{x}, \hat{u}) \in \mathcal{Z}_{\alpha, q}\left(t_{0}, T ; \mathcal{X}\right) \times \mathcal{U}_{\partial}$.

Proof. By Theorem 3 the set $\mathcal{W}$ of admissible pairs is nonempty. We use Theorem 2.4 from the monograph [24] for the proof of an optimal control existence. Take spaces $\mathbf{Y}:=\mathcal{Q}_{\alpha, q}\left(t_{0}, T ; \mathcal{X}\right)$, $\mathbf{Y}_{1}:=\mathcal{Z}_{\alpha, q}\left(t_{0}, T ; \mathcal{X}\right), \mathbf{U}:=L_{q}\left(t_{0}, T ; \mathcal{U}\right), \mathbf{V}:=L_{q}\left(t_{0}, T ; \mathcal{Y}\right) \times \mathcal{X}^{m}$, the operator $\mathbf{L}: \mathbf{Y}_{1} \times \mathbf{U} \rightarrow \mathbf{V}$ and the vector $\mathbf{F} \in \mathbf{V}$ of the form 


$$
\begin{gathered}
\mathbf{L}(x, u):= \\
=\left(L D^{\alpha} x-M x-\sum_{k=1}^{n} N_{k}(t) D_{t}^{\alpha_{k}} x-B u, \gamma_{0}(P x), \gamma_{0}(P x)^{(1)}, \ldots, \gamma_{0}(P x)^{(m-1)}\right), \\
\mathbf{F}:=-\left(0, x_{0}, x_{1}, \ldots, x_{m-1}\right) .
\end{gathered}
$$

The continuity of the linear operator $\mathbf{L}$ from $\mathcal{Z}_{\alpha, q}\left(t_{0}, T ; \mathcal{X}\right) \times L_{q}\left(t_{0}, T ; \mathcal{U}\right)$ to $L_{q}\left(t_{0}, T ; \mathcal{Y}\right) \times \mathcal{X}^{m}$ follows from the inequalities

$$
\begin{gathered}
\left\|\left(L D_{t}^{\alpha} x-M x-\sum_{k=1}^{n} N_{k}(t) D_{t}^{\alpha_{k}} x-B u, \gamma_{0}(P x), \gamma_{0}(P x)^{(1)}, \ldots, \gamma_{0}(P x)^{(m-1)}\right)\right\|_{L_{q}\left(t_{0}, T ; \mathcal{Y}\right) \times \mathcal{X}^{m}}= \\
=\left\|L D_{t}^{\alpha} x-M x-\sum_{k=1}^{n} N_{k}(t) D_{t}^{\alpha_{k}} x-B u\right\|_{L_{q}\left(t_{0}, T ; \mathcal{Y}\right)}+ \\
+\left\|\gamma_{0}(P x)\right\|_{\mathcal{X}}+\left\|\gamma_{0}(P x)^{(1)}\right\|_{\mathcal{X}}+\cdots+\left\|\gamma_{0}(P x)^{(m-1)}\right\|\left\|_{\mathcal{X}} \leq C_{L}\right\| D_{t}^{\alpha} x\left\|_{L_{q}\left(t_{0}, T ; \mathcal{X}\right)}+\right\| M x \|_{L_{q}\left(t_{0}, T ; \mathcal{Y}\right)}+ \\
+\sum_{k=1}^{n} C_{N_{k}}\left\|D_{t}^{\alpha_{k}} x\right\|_{L_{q}\left(t_{0}, T ; \mathcal{X}\right)}+C_{B}\|u\|_{L_{q}\left(t_{0}, T ; \mathcal{U}\right)}+C_{\gamma_{0} P}\|x\|_{C^{m-1}\left(\left[t_{0}, T\right] ; \mathcal{X}\right)} \leq \\
\leq C_{1}\left\|D_{t}^{\alpha} x\right\|_{L_{q}\left(t_{0}, T ; \mathcal{X}\right)}+C_{2}\|x\|_{L_{q}\left(t_{0}, T ; D_{M}\right)}+C_{3}\|x\|_{C^{m-1}\left(t_{0}, T ; \mathcal{X}\right)}+C_{4}\|u\|_{L_{2}\left(t_{0}, T ; \mathcal{U}\right)} \leq \\
\leq C\left(\|x\|_{\mathcal{Z}_{\alpha, q}\left(t_{0}, T ; \mathcal{X}\right)}+\|u\|_{L_{q}\left(t_{0}, T ; \mathcal{U}\right)}\right)=C\|(x, u)\|_{\mathcal{Z}_{\alpha, q}\left(t_{0}, T ; \mathcal{X}\right) \times L_{q}\left(t_{0}, T ; \mathcal{U}\right)} .
\end{gathered}
$$

Here we applied Lemma 1.

For a pair $(x, u) \in \mathcal{W}$ we have

$$
\begin{gathered}
\|(x, u)\|_{\mathbf{Y}_{1} \times \mathbf{U}}=\|x\|_{\mathcal{Z}_{\alpha, q}\left(t_{0}, T ; \mathcal{X}\right)}+\|u\|_{L_{q}\left(t_{0}, T ; \mathcal{U}\right)}= \\
=\|x\|_{L_{q}\left(t_{0}, T ; \mathcal{X}\right)}+\|M x\|_{L_{q}\left(t_{0}, T ; \mathcal{Y}\right)}+\|x\|_{C^{m-1}\left(t_{0}, T ; \mathcal{X}\right)}+\left\|D_{t}^{\alpha} x\right\|_{L_{q}\left(t_{0}, T ; \mathcal{X}\right)}+ \\
+\|u\|_{L_{q}\left(t_{0}, T ; \mathcal{U}\right)}=\|x\|_{L_{q}\left(t_{0}, T ; \mathcal{X}\right)}+\left\|L D_{t}^{\alpha} x-\sum_{k=1}^{n} N_{k} D_{t}^{\alpha_{k}} x-B u\right\|_{L_{q}\left(t_{0}, T ; \mathcal{Y}\right)}+ \\
+\|x\|_{C^{m-1}\left(t_{0}, T ; \mathcal{X}\right)}+\left\|D_{t}^{\alpha} x\right\|_{L_{q}\left(t_{0}, T ; \mathcal{X}\right)}+\|u\|_{L_{q}\left(t_{0}, T ; \mathcal{U}\right)} \leq \\
\leq C_{L}\left\|D_{t}^{\alpha} x\right\|_{L_{q}\left(t_{0}, T ; \mathcal{X}\right)}+C_{N}\|x\|_{C^{m-1}\left(\left[t_{0}, T\right] ; \mathcal{X}\right)}+C_{B}\|u\|_{L_{q}\left(t_{0}, T ; \mathcal{U}\right)} \leq C_{1} R+C_{2},
\end{gathered}
$$

if $J(x, u) \leq R$. Thus, functional (13) is coercive.

\section{Example}

Consider a control problem for the model equation

$$
\begin{gathered}
D_{t}^{\alpha}\left(\frac{\partial^{2} v}{\partial s^{2}}+\gamma v\right)=\beta v+\sum_{k=1}^{n} \delta_{k}(t) D_{t}^{\alpha_{k}}\left(\frac{\partial^{2} v}{\partial s^{2}}+\gamma v\right)+u, s \in(0, \pi), t \in\left(t_{0}, T\right), \\
v(0, t)=v(\pi, t)=0, \quad t \in\left(t_{0}, T\right), \\
\frac{\partial^{k}}{\partial t^{k}}\left(\frac{\partial^{2} v}{\partial s^{2}}+\gamma v\right)\left(s, t_{0}\right)=v_{k}(s), \quad k=0,1, \ldots, m-1, \quad s \in(0, \pi),
\end{gathered}
$$

$\beta, \gamma \in \mathbb{R}, \delta_{k}:\left(t_{0}, T\right) \rightarrow \mathbb{R}, k=1,2, \ldots, n, 0 \leq \alpha_{1}<\alpha_{2}<\cdots<\alpha_{n} \leq m-1<\alpha \leq m \in \mathbb{N}$. In order to reduce problem (14)-(16) to (8), (9) we choose spaces and operators: $\mathcal{X}=\left\{v: H^{2}(0, \pi): v(0)=\right.$ $v(\pi)=0\}, \mathcal{Y}=L_{2}(0, \pi), \mathcal{U}=L_{2}(0, \pi), L=\frac{\partial^{2}}{\partial s^{2}}+\gamma, M=\beta I, N_{k}(t)=\delta_{k}(t)\left(\frac{\partial^{2}}{\partial s^{2}}+\gamma\right), k=1,2, \ldots, n$, $B=I$. 
The admissible controls set and the cost functional are given by the following

$$
\begin{gathered}
\|u\|_{L_{2}\left(t_{0}, T ; L_{2}(0, \pi)\right)}^{2} \leq r^{2}, \\
J(v, u):=\left\|v-v_{d}\right\|_{C^{m-1}\left(\left[t_{0}, T\right] ; \mathcal{X}\right)}+\left\|D_{t}^{\alpha} v-D_{t}^{\alpha} v_{d}\right\|_{L_{2}\left(t_{0}, T ; \mathcal{X}\right)}^{2}+\delta\left\|u-u_{d}\right\|_{L_{2}\left(t_{0}, T ; L_{2}(0, \pi)\right)}^{2} \rightarrow \inf ,
\end{gathered}
$$

where $v_{d} \in C^{m-1}\left(\left[t_{0}, T\right] ; \mathcal{X}\right), D_{t}^{\alpha} v_{d} \in L_{2}\left(t_{0}, T ; \mathcal{X}\right), u_{d} \in L_{2}\left(t_{0}, T ; L_{2}(0, \pi)\right), r, \delta>0$.

Introduce the space

$$
\begin{gathered}
\mathcal{Z}_{\alpha, 2}\left(t_{0}, T ; \mathcal{X}\right):=\left\{v \in C^{m-1}\left(\left[t_{0}, T\right] ; \mathcal{X}\right) \cap L_{2}\left(t_{0}, T ; L_{2}(0, \pi)\right):\right. \\
\left.J_{t}^{m-\alpha}\left(v-\sum_{k=0}^{m-1} v^{(k)}\left(t_{0}\right) \tilde{g}_{k+1}\right) \in H^{m}\left(t_{0}, T ; \mathcal{X}\right)\right\} .
\end{gathered}
$$

Theorem 5. Let $m-\alpha<1 / 2, \gamma=b^{2}$ at some $b \in \mathbb{N}, v_{k} \in \mathcal{X}, k=0, \ldots, m-1$,

$$
\int_{0}^{\pi} v_{k}(s) \sin (b s) d s=0, \quad k=0,1, \ldots, m-1,
$$

$\delta_{l}:\left(t_{0}, T\right) \rightarrow \mathbb{R}$ are measurable and essentially bounded, $l=1,2, \ldots, n$. Then there exists a unique solution of problem (14)-(18) on $\left(t_{0}, T\right)$.

Proof. We have ker $L=\operatorname{span}\{\sin b s\}$, and Equation (14) is degenerate. The operator $M$ is $(L, 0)$-bounded, since for sufficiently large $|\mu|$ we have $\mu \neq \frac{\delta}{\lambda_{k}+\gamma}$ and

$$
\left\|(\mu L-M)^{-1} y\right\|_{H^{2}(\mathcal{X})}=\sum_{k=1}^{\infty} \frac{\left(1+\lambda_{k}^{2}\right)\left\langle y, \varphi_{k}\right\rangle^{2}}{\left(\mu\left(\lambda_{k}+\gamma\right)-\delta\right)^{2}} \leq C \sum_{k=1}^{\infty}\left\langle y, \varphi_{k}\right\rangle^{2} .
$$

This implies that $\operatorname{im} L=\mathcal{Y}^{1}$ and we can reduce problem (9) to the problem $(L x)^{(k)}\left(t_{0}\right)=v_{k}=$ $L x_{k} \in \mathcal{Y}^{1}, k=0,1, \ldots, m-1$, of form (16). Note that conditions (19) mean that $v_{k} \in \mathcal{Y}^{1}$. Moreover, $\operatorname{im}_{k}(t) \subset \operatorname{im} L=\mathcal{Y}^{1}$, since $N_{k}(t)=\delta_{k}(t) L, k=1,2, \ldots, n$.

We can take $u_{0}=0$ in the conditions of Theorem 4 . So by that theorem problem (14)-(18) has a unique solution.

\section{Conclusions}

We studied the unique solvability of initial value problems for a class of degenerate evolution fractional multi-term equations. The obtained results are applied to study of some optimal control problems for systems, which state is described by such initial value problem. Abstract results can be used for investigation of optimal control problems for multi-term time-fractional partial differential equations, it is illustrated on an example. The results of the work in future will be extended to problems with start control and with mixed control to degenerate evolution fractional multi-term equations, to stochastic degenerate fractional evolution equations with white noise, and some others.

Author Contributions: Conceptualization, M.P.; methodology, M.P.; validation, G.B.; formal analysis, G.B.; investigation, G.B.; writing-original draft preparation, G.B.; writing-review and editing, M.P.; supervision, M.P.; project administration, M.P. All authors have read and agreed to the published version of the manuscript.

Funding: The work is supported by Act 211 of Government of the Russian Federation, contract 02.A03.21.0011.

Conflicts of Interest: The authors declare no conflict of interest. The funders had no role in the design of the study; in the collection, analyses, or interpretation of data; in the writing of the manuscript, or in the decision to publish the results. 


\section{References}

1. Demidenko, G.V.; Uspenskii, S.V. Partial Differential Equations and Systems Not Solvable with Respect to the Highest-Order Derivative; Marcel Dekker, Inc.: New York, NY, USA; Basel, Switzerland, 2003.

2. Sviridyuk, G.A.; Fedorov, V.E. Linear Sobolev Type Equations and Degenerate Semigroups of Operators; VSP: Utrecht, The Netherlands; Boston, MA, USA, 2003.

3. Favini, A.; Yagi, A. Degenerate Differential Equations in Banach Spaces; Marcel Dekker, Inc.: New York, NY, USA; Basel, Switzerland; Hong Kong, China, 1999.

4. Baleanu, D.; Fedorov, V.E.; Gordievskikh, D.M.; Taş, K. Approximate controllability of infinite-dimensional degenerate fractional order systems in the sectorial case. Mathematics 2019, 7, 735. [CrossRef]

5. Baleanu, D.; Fernandez, A. On fractional operators and their classifications. Mathematics 2019, 7, 830. [CrossRef]

6. Fedorov, V.E.; Debbouche, A. A class of degenerate fractional evolution systems in Banach spaces. Differ. Equ. 2013, 49, 1569-1576. [CrossRef]

7. Fedorov, V.E.; Romanova, E.A.; Debbouche, A. Analytic in a sector resolving families of operators for degenerate evolution equations of a fractional order. J. Math. Sci. 2018, 228, 380-394. [CrossRef]

8. Shishkina, E.; Sitnik, S. A fractional equation with left-sided fractional Bessel derivatives of Gerasimov-Caputo type. Mathematics 2019, 7, 1216. [CrossRef]

9. Wang, J.R.; Zhou, Y. A class of fractional evolution equations and optimal controls. Nonlinear Anal. Real World Appl. 2011, 12, 262-272. [CrossRef]

10. Baleanu, D.; Machado, J.A.T.; Luo, A.C.J. Fractional Dynamics and Control; Springer: New York, NY, USA, 2012.

11. Dzielinski, A.; Czyronis, P.M. Optimal control problem for fractional dynamic dystems-Linear quadratic discrete-time case. Lect. Notes Electr. Eng. 2013, 257, 87-97.

12. Bahaa, G.M.; Hamiaz, A. Optimal control problem for coupled time-fractional diffusion systems with final observations. J. Taibah Univ. Sci. 2018, 13, 124-135. [CrossRef]

13. Plekhanova, M.V. Degenerate distributed control systems with fractional time derivative. Ural. Math. J. 2016, 2, 58-71. [CrossRef]

14. Plekhanova, M.V. Solvability of control problems for degenerate evolution equations of fractional order. Chelyabinsk Phys. Math. J. 2017, 2, 53-65.

15. Plekhanova, M.V. Distributed control problems for a class of degenerate semilinear evolution equations. J. Comput. Appl. Math. 2017, 312, 39-46. [CrossRef]

16. Plekhanova, M.V.; Baybulatova, G.D. Optimal control problems for a class of degenerate evolution equationswith delay. Chelyabinsk Phys. Math. J. 2018, 3, 319-331.

17. Plekhanova, M.V. Optimal control existence for degenerate infinite dimensional systems of fractional order. IFAC-PapersOnLine 2018, 51, 669-674. [CrossRef]

18. Plekhanova, M.V.; Baybulatova, G.D.; Davydov, P.N. Numerical solution of an optimal control problem for Oskolkov's system. Math. Methods Appl. Sci. 2018, 41, 9071-9080. [CrossRef]

19. Plekhanova, M.V.; Baybulatova, G.D. Problems of hard control for a class of degenerate fractional order evolution equations. Lect. Notes Comput. Sci. 2019, 11548, 501-512.

20. Plekhanova, M.V.; Baybulatova, G.D. Semilinear equations in Banach spaces with lower fractional derivatives. Springer Proc. Math. Stat. 2019, 292, 81-93.

21. Plekhanova, M.V.; Baybulatova, G.D. A class of semilinear degenerate equations with fractional lower order derivatives. In Stability, Control, Differential Games (SCDG2019), Proceedings of the International Conference Devoted to the 95th Anniversary of Academician N.N. Krasovskii, Yekaterinburg, Russia, 16-20 September 2019; Filippova, T.F., Maksimov, V.I., Tarasyev, A.M., Eds.; Krasovskii Institute of Mathematics and Mechanics of Ural Branch of the Russian Academy of Sciences (IMM UB RAS): Yekaterinburg, Russia, 2019; pp. 444-448.

22. Bajlekova, E.G. Fractional Evolution Equations in Banach Spaces. Ph.D. Thesis, Eindhoven University of Technology, Eindhoven, The Netherlands, 2001. 
23. Plekhanova, M.V.; Baybulatova, G.D. On strong solutions for a class of semilinear fractional degenerate evolution equations with lower fractional derivatives. Math. Methods Appl. Sci. 2020, in press.

24. Fursikov, A.V. Optimal Control of Distributed Systems. Theory and Applications; AMS: Providence, RI, USA, 1999.

(C) 2020 by the authors. Licensee MDPI, Basel, Switzerland. This article is an open access article distributed under the terms and conditions of the Creative Commons Attribution (CC BY) license (http://creativecommons.org/licenses/by/4.0/). 